\title{
Respostas às mudanças climáticas: inovação tecnológica ou mudança de comportamento individual?
}

\author{
FABIÁN ECHEGARAY ${ }^{I}$ \\ e MICHELE HARTMANN FEYH AFONSO II
}

\section{Introdução}

$\mathrm{P}$ oucos temas têm dominado o debate público e a agenda política nos últimos anos como as mudanças climáticas e o aquecimento global. Tanto nos meios de comunicação como entre as prioridades do público - e especialmente entre as preocupações -, o tema ganhou um espaço que não tinha, para além do nível de desenvolvimento, pressões ambientais, crise climática, ou padrão cultural das sociedades (Boykoff; Mansfield, 2008; Block, 2010).

De fato, em países emergentes como o Brasil o tema sextuplicou seu espaço na mídia, ${ }^{1}$ ao mesmo tempo que o número de eventos climáticos extremos ao redor do planeta se multiplicou de igual maneira à sua intensidade. Registros privados iniciados em 2004 indicavam a ocorrência de 640 eventos catastróficos, que deixaram como saldo 11.600 vítimas fatais e quase U\$ 108 bilhões em perdas; em 2010, os eventos somaram 960 com 67.800 vítimas fatais e U\$ 100 bilhões em perdas. ${ }^{2}$ Ainda, se no ano de 2000 o desvio da média de temperatura do século XX se limitou a 0,40 graus Celsius, em 2010 chegava a $0,63 .{ }^{3}$ Tanto as catástrofes como as mudanças abruptas na temperatura colocam o tema no radar e na realidade das sociedades (e não só das suas elites científicas ou ambientalistas), e facilitam tanto a visibilidade do tema como a formação de atitudes e opiniões sobre o assunto.

Como resultado, diversos estudos de opinião pública de caráter comercial como acadêmico têm revelado, de maneira sistemática, que parcelas expressivas das sociedades consideram o assunto dentro das prioridades da agenda nacional e - em comparação com outros temas - bastante grave ou sério. Na Europa, a grande maioria da população considera os perigos das mudanças climáticas um problema mais sério se comparado à crise financeira, ficando atrás apenas da pobreza. ${ }^{4}$ No mesmo sentido, uma ampla maioria de norte-americanos expressa ter sido afetada pessoalmente por eventos climáticos extremos nos últimos doze meses, associando essas ocorrências diretamente ao aquecimento global. ${ }^{5}$ 
A América Latina apresenta a mais alta taxa de preocupação com a questão das mudanças climáticas se comparada a outros grupos de países em uma pesquisa online, sendo quase a totalidade da população de internautas da região preocupada com os problemas decorrentes $(90 \%){ }^{6}$

O presente artigo busca identificar a tensão existente na opinião pública sobre as formas de encarar esse problema, isto é, enfocando nas mudanças pessoais dos estilos de vida ou privilegiando as mudanças externas resultantes de avanços tecnológicos, e, em segundo lugar, identificar a influência exercida pelas condições contextuais capazes de explicar as diferentes percepções entre as sociedades.

\section{As mudanças climáticas como problema}

Se tem havido um consenso ao redor das mudanças climáticas entre públicos tão diferentes como jornalistas, executivos de grandes corporações, cientistas, sindicatos, políticos, militantes sociais e escritores, de diversos setores, simpatias ideológicas e origens, é que se trata de um perigo iminente, assustador e de potencial devastador. $\mathrm{O}$ tom dramático, urgente e pessimista une segmentos de todo tipo. "Este é um livro sobre pesadelos, catástrofes" adverte o sociólogo Anthony Giddens (2010, p.19) em seu recente A política da mudança climática. "O aquecimento global deve ser visto como uma ameaça econômica e à nossa segurança", denuncia o ex-secretário da ONU, Kofi Annan. "As mudanças climáticas constituem um dos maiores desafios do nosso tempo", aponta a carta aberta do Instituto Ethos do Brasil, um think tank que reflete o pensamento empresarial do país sobre responsabilidade corporativa. ${ }^{7} \mathrm{O}$ líder social e ambientalista Lester Brown alerta que as mudanças climáticas significam menos comida e mais fome, e o seu livro Plano B apresenta como subtítulo "mobilização para salvar a civilização" (Brown, 2009).

Semelhante marco semântico quando veiculado pelos meios de comunicação de massa dificilmente passa despercebido pela opinião pública, consequentemente é de imaginar que o tema ganhe em visibilidade, intensidade de opiniões e sentido de urgência. De fato, já são $83 \%$ da população mundial que veem com gravidade a situação das mudanças climáticas ${ }^{8}$ reflexo da grande disseminação de informação e alarde sobre a questão conduzidos nos principais meios de comunicação e discussão.

Igualmente, na medida em que esses diferentes atores em razão de seus valores e interesses encaram diferentemente as alternativas válidas de ação, o equilíbrio entre dar preferência a mudanças nos estilos de vida versus a mudanças trazidas pela tecnologia acaba sendo afetado. Por exemplo, as empresas e alguns setores da comunidade científica e o governo (como ministérios de economia, planejamento, educação e tecnologia) trabalham com a premissa naturalizada de que o risco ou desafio não só representa uma situação de pressão como também um cenário de oportunidades. E boa parte dessas oportunidades está vinculada à geração e à oferta, por exemplo, de inovações em procedimentos e soluções tec- 
nológicas (tais como geradores de energia limpa, equipamentos que aumentam a eficiência energética, métodos verdes de construção civil etc.), o que inclinaria a balança a favor da preferência por saídas através da tecnologia (a promessa da "economia verde") (Unep, 2009; WBCSD, 2010). Por outro lado, as ONG e entidades da sociedade civil, outros setores do governo (como ministérios da saúde e meio ambiente), assim como inúmeros membros da comunidade acadêmica, questionam a eficácia das respostas tecnológicas e interpretam que o principal objetivo efetivo no longo prazo passa por modificar substancialmente as condutas pessoais e a aspiração a manter níveis de consumo insustentáveis, induzindo a uma preferência maior por mudar estilos de vida (Mont et al., 2012; Webb, 2012).

A tensão entre essas opções específicas também são nutridas por debates relativos a quão genuína é a predisposição dos principais afetados pelas mudanças climáticas (os indivíduos) a apropriar-se ou não do problema, pois essa escolha implica um alinhamento com estratégias que envolvem o indivíduo de maneira central. Até que ponto as respostas à crise ambiental devem vir de mudanças pessoais ou de mudanças externas impulsionadas por inovações tecnológicas se constitui, assim, em um indicador-chave da intensidade dos compromissos pessoais com o tema, do sentido de responsabilização vigente e da percepção de empoderamento individual existente.

\section{A tecnologia como solução}

Soluções de eficiência energética que reduzam o consumo de água e energia e, portanto, as emissões de gases de efeito estufa; soluções de reciclagem de resíduos sólidos; procedimentos de despoluição de matérias-primas como água (Brown, 2009); e diversificação da matriz energética, mediante adoção de fontes renováveis de energia (Lund, 2007) compõem a primeira onda de inovações tecnológicas na busca por adaptação às mudanças climáticas. Atualmente, se somam a esses recursos tecnologias mais avançadas como a captura e armazenamento geológico de dióxido de carbono (Holloway, 2007); a nanotecnologia, que permite a manipulação de matéria em escala nanométrica; a biologia sintética, que permite a manipulação de elementos do DNA de organismos vivos; e a geoengenharia, que intervém em grande escala em oceanos e na atmosfera (Bermann, 2012).

A escolha entre um caminho através de mudanças pessoais ou de mudanças externas também reflete parte das contradições naturais da modernidade. Nesse sentido, a fé na saída tecnológica é uma derivação natural da crença moderna que consagra o domínio racional das atividades e processos (Beck, 1992; Beriain, 1995). A tecnologia é mais que um instrumento, ela constitui evidência manifesta do êxito da racionalização das ações e da organização hierárquica e estruturante das respostas aos problemas. Por outro lado, a crença em uma maior contribuição resolutiva das mudanças de estilo de vida também abriga um questionamento tácito sobre delegar às estruturas de decisão (governo, em- 
presas, comunidade científica) o monopólio da ação e da credibilidade em sua efetiva capacidade de implementar procedimentos operacionais e técnicos, ou de controlar que as aplicações tecnológicas sigam os standards de segurança e qualidade prescritos. Por último, tal preferência também reconhece dúvidas sobre as virtudes da tecnologia e admite a dimensão do risco associada a tal tecnologia (Beck, 1992).

$\mathrm{Na}$ literatura, essa percepção de risco pode surgir tanto do reconhecimento de limitações ou de impactos inócuos da tecnologia ao lidar com determinados problemas como de seus efeitos colaterais nocivos e consequências perigosas não esperadas. Assim, a admissão de que as mudanças de estilo de vida e padrões de comportamento pessoal são formas mais efetivas de combater as mudanças climáticas do que as mudanças tecnológicas pode estar abrigando um ceticismo aberto a respeito destas últimas (Beck, 1992; Beriain, 1995).

Por outro lado, a inovação tecnológica é tratada como parte importante do processo de adaptação às mudanças climáticas (Hertin et al., 2002; Adger et al., 2009), ou mesmo como parte central desse desafio (Winkler; Marquand, 2009). Muito embora o desenvolvimento tecnológico não seja suficiente para lidar com o problema como um todo, havendo necessidade de trabalhar também questões sociais e culturais (Rajan, 2006), a falta de acesso à tecnologia se traduz em uma forte barreira na busca pela adaptação (Scheraga; Grambsch, 1998).

Nesse contexto, surgem duas perguntas sobre as quais concentraremos nossa análise. Considerando a centralidade das mudanças climáticas como problema da agenda pública: qual é a alternativa preferida de solução entre a aposta na mudança tecnológica e a mudança nos estilos de vida? E quais fatores estão influenciando essa escolha? Concretamente, que peso têm as variáveis de desenvolvimento socioeconômico, de maturidade institucional e de tensão ambiental sobre a escolha de uma saída mais tecnológica ou mais comportamental à crise ambiental atual? O estudo dos fatores de influência da inclinação para uma solução mais tecnológica em detrimento de uma mudança de hábitos individuais em relação às mudanças climáticas será investigado sob três hipóteses, as quais são descritas a seguir.

$\mathrm{Hl}$ : O grau de desenvolvimento socioeconômico está negativamente associado com a crença na opção tecnológica como solução para o problema das mudanças climáticas, estimulando mudanças nos hábitos de vida individuais.

A condição econômica é tida como determinante na maneira como os países lidam com as mudanças climáticas (Kim; Choi, 2011). No nível macro, sabe-se que a população residente em países mais ricos possui maior vivência com o avanço tecnológico e com os perigos decorrentes. As inovações que permitiram acelerar o crescimento econômico, acumular bens e garantir bem-estar geraram impactos sociais e ambientais cada vez menos ignorados como "externalidades" e cada vez mais visíveis como riscos ou problemas (Jackson, 2009). Essa experiência tem levado a um posicionamento menos favorável à tecnologia, eviden- 
ciando uma relação negativa entre a riqueza da nação e o apoio à tecnologia (Bauer; Durant; Evans, 1994).

Quando o foco recai sobre as nações em desenvolvimento, é reconhecido que a falta de recursos está diretamente relacionada com a vulnerabilidade (Chan; Parker, 1996; Fankhauser; Tol, 1997). Embora a pobreza não possa ser entendida como um sinônimo de vulnerabilidade, é tida como um indicador aproximado da capacidade de lidar com o problema (Dow, 1992). Nesses países, observa-se que os indivíduos tendem a apresentar uma percepção de risco mais acentuada da situação, se tornando, portanto, mais propensos a depositarem suas expectativas sobre a tecnologia e negando os perigos que poderiam estar associados à essa opção, uma vez que possuem poucos recursos para se mobilizarem de outras formas (Graham; Chang; Evans, 1992).

Nesse contexto, busca-se verificar se o grau de desenvolvimento econômico inibe a expectativa sobre a tecnologia como solução para o problema das mudanças climáticas, aumentando a propensão a se obter mudanças nos hábitos individuais.

$\mathrm{H} 2$ : O grau de desenvolvimento institucional está positivamente associado com a crença na opção tecnológica como solução para o problema das mudanças climáticas, inibindo mudanças nos hábitos de vida individuais.

As instituições fornecem o contexto organizacional e sociocultural no qual as atividades humanas são estruturadas, os valores são expressos e as normas são criadas (Burch, 2011). Elas representam os sistemas de regras que direcionam a tomada de decisões e a lógica subjacente para as organizações (Burch, 2011), e têm o papel de manter a sociedade coesa, atribuindo-lhe sentido e finalidade, e permitindo-lhe adaptar-se (O'Riordan; Jordan, 1999). Dessa forma, a questão das mudanças climáticas se relaciona fortemente com o grau de maturidade institucional, uma vez que a seriedade atribuída ao problema deriva do modo como a sociedade está organizada, sua relação com a base de recursos, sua relação com outras sociedades e as relações entre seus membros (Rayner; Malone, 1998).

De forma geral, países que possuem instituições sociais mais desenvolvidas (por exemplo, aqueles que se apoiam na autorregulação ou na regulação após deliberação, permitem a livre circulação de ideias e propostas, estimulam o surgimento e consolidação de práticas empresariais responsáveis que levam em conta todos os custos de produção de bens, comunicam livremente os impactos positivos e negativos da atuação corporativa sobre a sociedade e meio ambiente etc.) tendem a apresentar maior capacidade de adaptação às mudanças climáticas do que aqueles cuja organização institucional é menos evoluída, o que ocorre comumente nos países emergentes (Smith; Lenhart, 1996). A falta de suporte institucional é geralmente vista como um obstáculo para a adoção de políticas de adaptação, sendo os países desenvolvidos, os quais apresentam uma base institucional mais sólida, que apresentam maior facilidade para lidar com os problemas enfrentados e também com riscos futuros (Smit; Pilifosov, 2001). 
As capacidades institucionais exibidas por uma sociedade influenciam na abordagem de problemas sociais como as mudanças climáticas, tendendo a favorecer a utilização de tecnologias. Sociedades abertas e empresas trilhando o caminho da sustentabilidade de forma mais consistente encontram limitações para socializar os custos de absorver medidas que afetem os interesses das suas clientelas, como eleitores e consumidores (Gourevitch, 1993; Hoffman, 2007). Nesse sentido, ambas as condições institucionais tenderiam a favorecer respostas que descansem menos na internalização de custos maiores entre seus públicos de relacionamento (tais como a mudança de hábitos e estilos de vida) e descansam mais em opções menos sujeitas à contestação como os avanços tecnológicos. Nessa perspectiva, busca-se verificar se nações mais bem estruturadas institucionalmente, e, portanto, com maiores chances de terem vivenciado experiências em termos de inovação tecnológica, proporcionariam maior aceitação da tecnologia como caminho de solução para as mudanças climáticas.

H3: As condições ambientais favoráveis que caracterizam uma sociedade estão positivamente associadas com a crença na opção tecnológica como solução para o problema das mudanças climáticas, inibindo mudanças nos hábitos de vida individuais.

Reações da opinião pública diante do problema das mudanças climáticas tendem a ser influenciadas pelo contexto no qual essa população está inserida. Trabalhos anteriores indicam que, diante de situações em que os riscos das mudanças climáticas não sejam percebidos, a tendência é de que nenhuma ação de adaptação seja tomada (como verificado na pesquisa de O'Brien et al. (2006) na Noruega). Dessa forma, a mobilização para ações de adaptação depende do conhecimento e antecipação de impactos locais e das percepções dos indivíduos, considerando a brecha cognitivo-comportamental existente entre conhecimento dos impactos, valores, crenças, normas e ações (Jackson, 2005).

Ainda que a adesão a ações de adaptação dependa de percepções individuais e conhecimento do contexto local, não é claro de que forma os impactos vivenciados no passado influenciam o posicionamento perante a questão para o futuro. Observa-se que nos países desenvolvidos as alterações climáticas e os impactos decorrentes, apesar de gerarem preocupação, parecem ser subestimados ou negligenciados (Adger et al., 2009). Os indivíduos falham em estabelecer a conexão entre eventos climáticos extremos vivenciados no passado com possíveis ocorrências futuras similares (Whitmarsh, 2008), além de existir uma assimilação enviesada da questão onde somente são retidas as informações que estiverem de acordo com crenças preexistentes (Lord; Ross; Lepper, 1979; Rachlinski, 2000).

Nesse contexto de limitado engajamento individual em ações de adaptação às mudanças climáticas, até mesmo entre grupos que já vivenciaram impactos decorrentes, busca-se verificar se nações com melhores condições ambientais estariam menos propensas a incorporar mudanças nos hábitos individuais, atribuindo maior confiança na tecnologia como solução para o problema. 


\section{Metodologia}

O presente trabalho é construído com base em dados primários e secundários, que nos permitem buscar respostas para as perguntas apresentadas anteriormente. Entre os dados primários aparece a preferência por inovações tecnológicas ou por alterações no estilo de vida como forma de solução. Esses dados provêm do estudo anual de opinião pública Radar, da rede GlobeScan, referente ao período de 2007 a 2011, com medições bianuais, englobando quinze países, para os quais são realizadas 14.150 entrevistas em cada medição. Esses países incluem Alemanha, Brasil, Chile, China, Espanha, Estados Unidos, Filipinas, Grã-Bretanha, Índia, Indonésia, México, Nigéria, Quênia, Rússia e Turquia. Em cada país, institutos de pesquisa independentes implementaram o mesmo conjunto de perguntas para amostras representativas nacionais ou urbanas de adultos de todas as classes e ambos os sexos. ${ }^{9}$

Uma série de indicadores secundários foi analisada com o objetivo de testar as hipóteses formuladas. Para testar a primeira hipótese foram procurados indicadores estruturais dos países estudados; para a segunda hipótese, indicadores institucionais; e para a terceira, indicadores ambientais. Para cada uma dessas dimensões de análise, buscou-se encontrar um a dois indicadores secundários capazes de representá-las na investigação de influência dessas características das nações sobre a crença da população de que inovações tecnológicas possam resolver o problema das mudanças climáticas.

A partir de um grupo variado de indicadores investigados, optou-se por representar cada dimensão pelos seguintes indicadores: para a dimensão estrutural será utilizado o Índice de Desenvolvimento Humano (IDH), calculado pela Organização das Nações Unidas (ONU); para a dimensão institucional serão utilizados os indicadores de contagem de empresas publicando relatórios de sustentabilidade no formato GRI, fornecido pela Global Report Initiative, e o Índice de Liberdade, desenvolvido pela Heritage Foundation; e para a dimensão ambiental será utilizado o Índice de Desenvolvimento Ambiental (EPI Index), calculado pela Universidade de Yale (a descrição de cada indicador é apresentada no Anexo). Outros indicadores investigados foram descartados por apresentar problemas, tais como: dados incompletos para os países e medições ao longo do tempo considerados neste trabalho, mínima variabilidade entre países e/ou medições ao longo do tempo; problemas de colinearidade com outras variáveis consideradas. ${ }^{10}$ Adicionalmente, foi explorada uma dimensão psicológica, incluindo indicadores de percepção individual dos problemas das mudanças climáticas originados em estudos de opinião pública, que foi desconsiderada devido à inexistência de mais do que um indicador disponível para operacionalizar a medida, o qual apresentou mínima variabilidade ao longo das medições.

Os dados primários e secundários descritos estão disponíveis para quinze países em três unidades de tempo, totalizando 45 unidades de análise. Os dados 
primários foram coletados nos anos 2007, 2009 e 2011. Como a opinião pública reflete reações a experiências ou observações de realidades já estabelecidas ou anunciadas, os dados secundários correspondem a uma unidade de tempo anterior, consistindo nos anos 2006, 2008 e 2010.

Dada a natureza incipiente do debate empírico sobre como entender as oscilações do humor público em torno desse assunto e de que maneira identificar e delimitar os seus determinantes, a nossa abordagem será inicialmente de caráter exploratório, verificando a intensidade e a direção das associações entre $\mathrm{o}$ indicador primário e os secundários mencionados para, em seguida, aprofundar no tipo das relações presentes. Para isso, utilizamos gráficos de frequências, análises de variância e modelo de efeitos aleatórios para dados em painel, que combinam corte transversal e séries temporais.

\section{Resultados}

Com altos e baixos, a questão da mudança climática tem ocupado um espaço significativo como parte da agenda nacional para a maioria dos cidadãos do mundo na última década. Pelo menos $51 \%$ dos países desenvolvidos e $64 \%$ dos países emergentes em 2011 expressavam um alto grau de preocupação com o tema (Gráfico 1) - entre os latino-americanos esse percentual chegava a 78\%.

Esses níveis de preocupação, no entanto, têm seguido caminhos diferentes. Entre os países de primeiro mundo, o senso de urgência começou mais cedo; assim, em 1998, o percentual dos que entendiam o problema das mudanças climáticas como muito grave era quase o dobro do observado em países emergentes. Depois de um pico de instabilidade em 2009, fruto da mobilização internacional em torno da COP-15 de Copenhague, o alarme dos europeus e norte-americanos sobre essa questão diminuiu ligeiramente, embora com oscilações. Por outro lado, entre os países em desenvolvimento, a comparação das opiniões em 1998 e 2007, aponta a duplicação do desconforto, seguindo nos anos seguintes as tendências voláteis dos países industrializados, embora com uma recuperação mais acentuada dos níveis de ansiedade ao redor do tema.

\section{A crença na tecnologia}

A esperança de que o problema das mudanças climáticas possa ser solucionado pelo desenvolvimento tecnológico, dispensando ou reduzindo a necessidade de mudanças no pensamento humano e no comportamento individual, é compartilhada por grande parte da população ao redor do globo: metade da população vê na tecnologia uma solução para o problema (Grafico 2). Ainda que elevado, o grupo de pessoas com fé na tecnologia vem se reduzindo e apresenta uma queda de dez pontos percentuais em um período de quatro anos (2007 a 2011), sinalizando uma percepção de maior necessidade do envolvimento individual com o problema.

As nações desenvolvidas se distanciam bastante da média global no sentido de não depositar tanto a esperança de solução do problema das mudanças climáticas sobre a tecnologia, e essa posição se acentua de forma mais acelerada 
ao longo do tempo, apresentando uma queda de dezoito pontos percentuais no grupo que deposita suas esperanças sobre a tecnologia (52\% para $34 \%$ ). Os países emergentes, pelo contrário, se mostram mais confiantes na tecnologia como caminho de solução para a questão, sendo essa a opinião de mais da metade da população desses países. Mas essa confiança mais elevada não impede o comportamento de queda observado nos outros grupos de países.

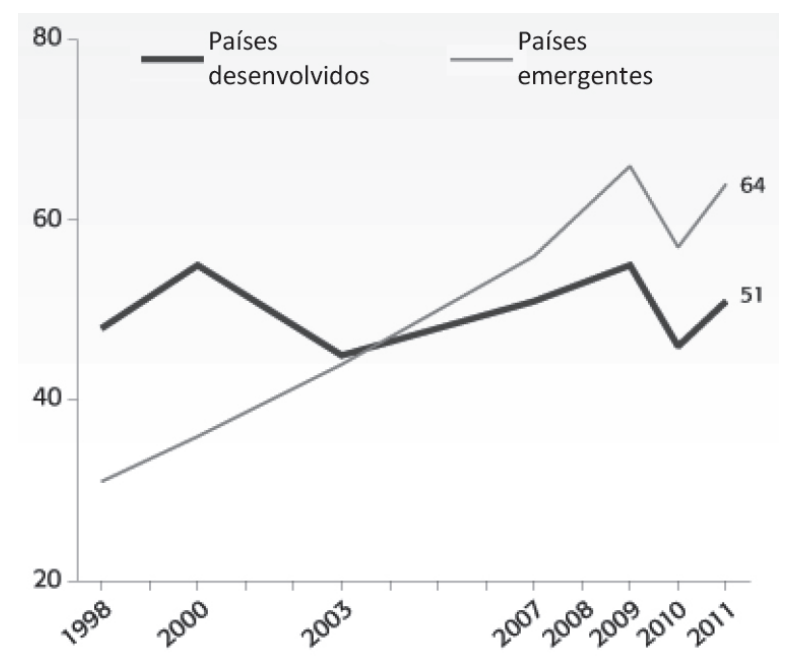

Fonte: Elaborado pelos autores com base em GlobeScan (2011).

Gráfico 1 - Gravidade dos problemas ambientais: mudanças climáticas (percentual de "problema muito sério", Países desenvolvidos versus Países emergentes, 1998-2011).

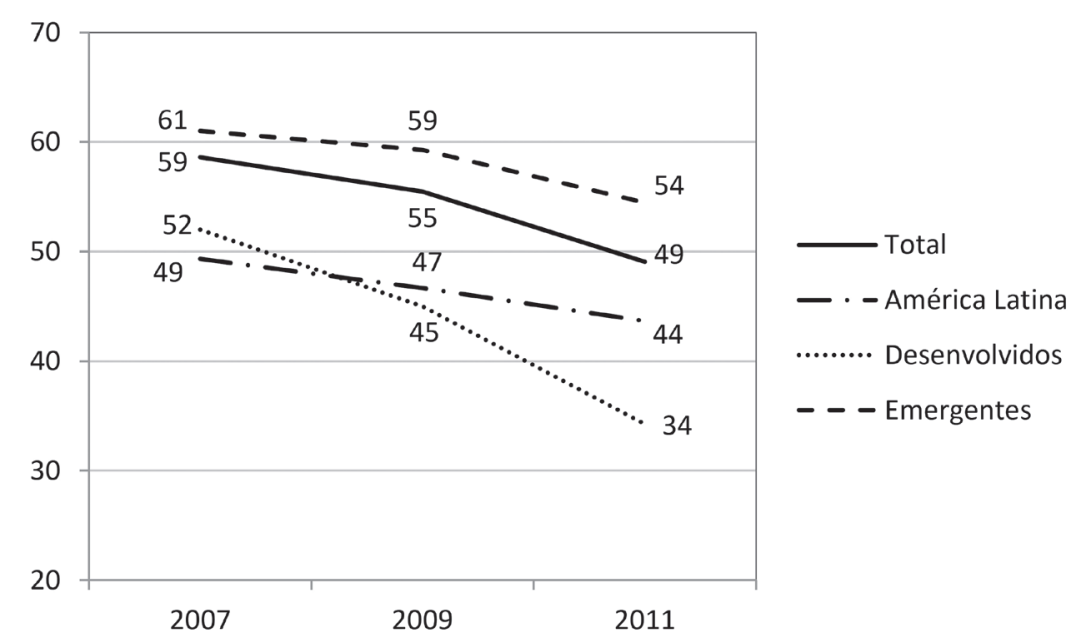

Fonte: Elaborado pelos autores com base em GlobeScan (2011).

Gráfico 2 - Crença na tecnologia como solução para o problema das mudanças climáticas - percentual de pessoas que concorda com a proposição. "Novas tecnologias resolverão o problema das mudanças climáticas, exigindo mudanças pequenas no pensamento e comportamento humano." 
O grupo formado por países da América Latina se destaca por apresentar uma posição mais estável ao longo do tempo. Distante do grupo de países emergentes (do qual fazem parte), os países da América Latina se mostram mais céticos com relação à tecnologia desde 2007 e essa posição se mantém relativamente estável, não apresentando uma queda tão acentuada quanto a observada em outros grupos de países.

\section{Os determinantes da fé na tecnologia}

A busca de entendimento sobre os fatores impulsionadores de uma atitude mais esperançosa acerca da tecnologia como solução para o problema das mudanças climáticas foi estudada sob três dimensões: estrutural, institucional e ambiental, para quinze países e em três unidades de tempo. Os indicadores selecionados para análise são apresentados na Tabela 1 com as suas estatísticas descritivas, revelando as médias e variabilidades obtidas para as medições nos países e unidades de tempo pesquisadas.

Tabela 1 - Estatísticas descritivas dos indicadores secundários estudados

\begin{tabular}{l|l|c|c|c|c}
\hline Dimensão & Indicadores & Mínimo & Máximo & Média & $\begin{array}{c}\text { Desvio } \\
\text { Padrão }\end{array}$ \\
\hline Estrutural & IDH & 0,4 & 0,9 & 0,7 & 0,1 \\
\hline Institucional & GRI & 0 & 184 & 35 & 47 \\
\hline Institucional & Freedom Index & 48,7 & 89,9 & 62,6 & 11,0 \\
\hline Ambiental & EPI Index & 40,2 & 86,3 & 68,3 & 12,8 \\
\hline
\end{tabular}

Fonte: Elaborado pelos autores com base em GlobeScan (2011).

Uma análise da variabilidade das questões de interesse entre os diferentes países e períodos estudados aponta que a principal fonte de variabilidade nos dados é proveniente da característica espacial, ou seja, os diferentes países estudados explicam uma grande parcela da variação observada nos dados (Tabela 2). O tempo, por outro lado, é responsável por uma pequena parcela da variabilidade em algumas variáveis específicas: na quantidade de empresas publicando relatórios de sustentabilidade no formato GRI e no indicador de desempenho ambiental.

Esse resultado indica que características mais estruturais, tais como desenvolvimento humano, e características institucionais como a liberdade de uma nação tendem a se manter estáveis em cada país para o curto espaço de tempo analisado. Mas o engajamento corporativo em ações de sustentabilidade (mensurado pela quantidade de publicações de relatórios de sustentabilidade no formato GRI) e o desempenho ambiental das nações (mensurado pelo EPI Index) enfrentam algumas transformações nesse espaço de tempo, evidenciando uma movimentação de diferentes nações na maneira como lidam com a questão da sustentabilidade em diferentes esferas da sociedade. 
Tabela 2 - Análise de variância através do tempo e do espaço - Proporção da variância total explicada pelo tempo e pelo espaço

\begin{tabular}{|c|c|c|c|c|}
\hline & & \\
\hline & & Ano & País & $\mathrm{N}$ \\
\hline Dependente & $\begin{array}{l}\text { Confiança na } \\
\text { tecnologia }\end{array}$ & 6,90 & $78,70^{\star *}$ & 45 \\
\hline Estrutural & $\mathrm{IDH}$ & 0,31 & $99,60^{\star \star}$ & 45 \\
\hline \multirow[t]{2}{*}{ Institucional } & GRI & $11,17^{\star}$ & $74,10^{\star \star}$ & 45 \\
\hline & FREE & 0,02 & $91,60^{\star *}$ & 45 \\
\hline Ambiental & EPI & $24,80^{* \star}$ & $70,90^{\star \star}$ & 45 \\
\hline
\end{tabular}

Fonte: Elaborado pelos autores com base em GlobeScan (2011).

${ }^{*} \mathrm{p}<0,10$ e ${ }^{* *} \mathrm{p}<0,05$ no teste $\mathrm{F}$.

Para entender o que leva a que as diferentes sociedades tendam a privilegiar mais ou menos a tecnologia como solução para o problema das mudanças climáticas ante as mudanças no comportamento humano, e entender também com que intensidade isso acontece, foi construído um modelo multivariado para dados de painel que incorpora informação sobre a influência de cada uma das três esferas apresentadas (estrutural, institucional e ambiental) na questão em estudo. ${ }^{11} \mathrm{~A}$ seleção das variáveis representativas de cada dimensão no modelo foi realizada conforme descrito na seção de Metodologia, e o modelo obtido é apresentado na Tabela $3 .^{12}$

Retomando as três hipóteses desenvolvidas na seção de Introdução, verifica-se que quanto maior é o Índice de Desenvolvimento Humano de uma nação (IDH), menor é a expectativa sobre a tecnologia como solução para o problema das mudanças climáticas. Esse resultado confirma a primeira hipótese desenvolvida, a qual estabelece que o grau de desenvolvimento socioeconômico está negativamente associado com a crença na opção tecnológica como solução para o problema das mudanças climáticas, estimulando mudanças nos hábitos de vida individuais.

Esses resultados convergem com estudos anteriores (Bauer; Durant; Evans, 1994; Slovic, 1999; Dosman; Adamowicz; Hrudey, 2001), indicando que diante de novos fenômenos como as mudanças climáticas, as quais são tidas como produto do crescimento econômico e expansão do bem-estar, o ceticismo tecnológico aumenta proporcionalmente ao grau de exposição ao desenvolvimento material. Como consequência, essa maior familiaridade com a tecnologia pode levar a um efeito de conscientização das limitações da promessa tecnológica e de ceticismo ante propostas de economia verde, pelo menos se reduzidas apenas ao conceito de produzir e consumir bens tecnologicamente superiores em termos de pegada ecológica. 
Tabela 3 - Modelo de efeitos aleatórios com estimação de máxima verossimilhança

\begin{tabular}{lcc}
\cline { 2 - 3 } & Coeficiente & Erro Padrão \\
\hline IDH & $-2,24776^{* *}$ & 0,522 \\
GRI & $0,00460^{*}$ & 0,002 \\
GRI.2 & $-0,00003^{* *}$ & 0,000 \\
FREE & 0,00223 & 0,005 \\
EPI & $0,00602^{* *}$ & 0,003 \\
Constante & $4,92860^{* *}$ & 0,262 \\
\hline sigma u & $0,14780^{* *}$ & 0,041 \\
sigma e & $0,13472^{\star *}$ & 0,019 \\
\hline $\mathrm{N}$ & 45 & \\
$\mathrm{~N}$ (peinéis) & 15 &
\end{tabular}

Wald Chi2 43,020**

rho $\quad 0,546$

Fonte: Elaborado pelos autores com base em GlobeScan (2011).

${ }^{*} p<0,05{ }^{* *} p<0,01$

O desenvolvimento institucional de uma nação impacta de forma mais complexa na expectativa da população sobre a tecnologia como caminho para enfrentar as mudanças climáticas. Existe um umbral no qual a maturidade institucional, medida pela quantidade de empresas publicando relatórios no formato GRI (GRI), favorece a fé na tecnologia. Mas, atingido certo nível de desenvolvimento institucional, essa relação se inverte, revelando críticas à solução tecnológica nas sociedades mais avançadas em termos de expressão e estruturação corporativa sustentável, como é possível evidenciar a partir da relação negativa com a medida de GRI elevada ao quadrado (GRI.2).

Por outro lado, o indicador de liberdade das nações indica que quanto maior é a liberdade de uma nação, maior é a esperança de que a tecnologia possa solucionar problemas decorrentes das mudanças climáticas, embora não tenha atingido significância no modelo construído. Ainda que reflita a dimensão institucional, esse indicador difere do anterior (GRI) em termos de significado, uma vez que reflete mais os modelos de relações institucionais no país, enquanto o indicador GRI aponta para a mobilização corporativa espontânea em torno das 
questões de sustentabilidade. Da mesma forma, esse efeito peculiar em sociedades com liberdades garantidas, mas não reguladas por um amadurecimento empresarial diante da sustentabilidade, sugere também a existência de um modelo de crença exclusiva na capacidade do mercado por reverter seus próprios problemas antes que de confiança nas iniciativas de adaptação social ou comportamental.

Assim, a hipótese de que o grau de institucionalização de uma nação esteja positivamente associado à crença tecnológica como solução para o problema das mudanças climáticas é endossada para nações até certo grau de desenvolvimento institucional, a partir do qual passa-se a aceitar a opção de adotar mudanças como comportamento individual como alternativa para o problema. São exemplos dessa relação os Estados Unidos e a Espanha, países com as mais elevadas taxas de publicação de relatórios de sustentabilidade no formato GRI e população pouco esperançosa sobre soluções tecnológicas para o problema das mudanças climáticas.

A terceira hipótese, que relaciona o estágio de desempenho ambiental das nações com o caminho de solução para o problema das mudanças climáticas, revela que as nações que estão em melhores condições ambientais são aquelas que mais depositam fé sobre a tecnologia para solucionar futuras questões acerca das mudanças climáticas. Esse resultado é consistente com estudos anteriores (O'Brien et al., 2006) e confirma a hipótese formulada, a qual se baseia sobre o pressuposto de que quanto menor é o envolvimento e a vivência dos indivíduos com situações ambientais precárias, menor será a disposição para mudanças no comportamento individual como forma de solução para o problema.

De forma geral, observa-se um bom ajuste do modelo, como é verificado pelo teste de Wald e do coeficiente rho, o qual sugere que o modelo utilizado é capaz de reduzir em mais da metade o erro em explicar a variabilidade da variável dependente. Dessa forma, as variáveis independentes consideradas se mostram capazes de reduzir incertezas acerca da preferência por soluções tecnológicas para o problema do câmbio climático. Outra forma de verificar a eficácia do modelo é por meio dos valores preditos para alguns casos que participaram do estudo. Ao se observar, por exemplo, os Estados Unidos, nação que menor expectativa deposita sobre a tecnologia em 2011, verifica-se um percentual observado de $29 \%$ da população com fé na tecnologia versus $27 \%$ predito pelo modelo. No extremo oposto, observa-se a Nigéria, com valor observado de 76\% da população que acredita tecnologia em 2011 , ante $72 \%$ predito pelo modelo.

\section{Conclusões}

Reflexões sobre as mudanças climáticas e o aquecimento global cada vez mais deixam de fazer parte somente das discussões acadêmicas e debates políticos para se tornarem preocupações concretas dos cidadãos em diversos países do globo. A população dos países emergentes é a que mais se mostra alarmada com a questão atualmente, refletindo um grande crescimento da preocupação com o tema nas últimas décadas nesses países. 
Este trabalho buscou identificar a tensão existente ao interior da opinião pública entre a crença na inovação tecnológica como solução para o problema das mudanças climáticas ante a alternativa de incorporar mudanças no comportamento individual, bem como identificar os determinantes desse posicionamento para um grupo diversificado de países em termos de desenvolvimento e posição geográfica.

Os resultados mostram que a inovação tecnológica é uma solução considerada por uma grande parcela da população em diversos países como caminho para solucionar o problema das mudanças climáticas. Mas a crença nessa opção já foi mais acentuada em anos anteriores, estando atualmente em queda e abrindo espaço para a percepção de necessidade de mudanças nos estilos de vida das pessoas. Os países desenvolvidos são os precursores desse movimento, enquanto os emergentes ainda mantêm expectativas mais elevadas sobre as soluções tecnológicas.

A investigação dos determinantes da fé na tecnologia como solução para o problema das mudanças climáticas revela que são os países menos desenvolvidos, com moderado desenvolvimento institucional e condições ambientais favoráveis que suportam a solução do problema sobre a tecnologia. Enquanto países mais desenvolvidos, com forte desenvolvimento institucional e condições ambientais desfavoráveis, se mostram mais céticos com relação à tecnologia, percebendo como necessárias mudanças nos hábitos e comportamentos individuais.

Esse cenário revela certa fragilidade das soluções tecnológicas como opção para lidar com as mudanças climáticas, a qual pode ser evidenciada sob diversos aspectos. Por um lado, observa-se a população de países desenvolvidos, a qual naturalmente tem mais acesso à tecnologia, apresentando baixa credibilidade sobre esse caminho de solução. Adicionalmente, essa é também a posição observada para a população de países com forte desenvolvimento institucional, condição que simboliza, inclusive, maior engajamento corporativo em termos de discussão e exposição pública de iniciativas adotadas acerca da sustentabilidade e medidas de mitigação das mudanças climáticas. Por outro lado, tem-se a população de países com condições ambientais desfavoráveis, a qual possui maior legitimidade para opinar sobre o assunto, e que também percebe de forma menos efetiva o enfrentamento do problema por meio de soluções tecnológicas.

Essa descrença sobre a tecnologia para o público que é considerado mais envolvido com o tema, seja pela vivência com os problemas ambientais diretamente, seja pelo maior acesso à tecnologia, ou mesmo pela possibilidade de acompanhar discussões institucionais e corporativas acerca do tema, revela que a opção por soluções tecnológicas pode ser considerada um caminho inicial, emergencial ou complementar para o problema, se mostrando pouco eficiente a longo prazo se considerada individualmente e revelando a necessidade de transformações mais profundas, tais como mudanças nos hábitos e comportamentos individuais da população. 
O posicionamento das nações mais desenvolvidas em acreditar que a solução para o problema das mudanças climáticas requer, de fato, mudanças no comportamento individual pode indicar um importante caminho de ação para as sociedades em desenvolvimento. Não é raro que essas nações apostem em conciliar a expansão material e econômica com respostas efetivas para o problema das mudanças climáticas através do investimento e da inovação em tecnologia. Sem embargo, a experiência vivenciada no primeiro mundo pode ser tomada como exemplo para alertar sobre os efeitos negativos dessa abordagem (contaminação, consumismo, estresse material etc.) e para reforçar a legitimidade de estilos de vida não ancorados na acumulação de bens.

Ainda assim, a inovação tecnológica está longe de poder ser desprezada no processo de mitigação das mudanças climáticas, uma vez que o caminho de enfrentamento do problema exige a busca por soluções de desafios complexos nos campos de produção, consumo, descarte e uso de recursos naturais. Adicionalmente, a adoção de soluções tecnológicas no combate as mudanças climáticas pode ser um primeiro passo para as nações em que outras transformações possam ser mais difíceis de serem alcançadas ou que não possam ser consideradas no curto prazo.

\section{Notas}

1 Por exemplo, segundo arquivos do principal jornal do Brasil, Folha de S.Paulo, a média de noticias sobre esse assunto saltou de 129 entre os anos 2000-2001 para 919 entre 2009-2010, com um crescimento bastante linear durante os anos intermediários.

2 Munich Re, NatCat service, 2011. Os dados de vítimas fatais e perdas consideram somente os resultantes de eventos climatológicos, hidrológicos e meteorológicos, e excluem os vinculados a fenômenos geológicos naturais como os vulcanológicos, terremotos e tsunamis.

3 NOAA, National climatic data center, US Dept of Commerce, 2011.

4 Pesquisa Eurobarometer, realizada pela TNS Opinion \& Social. Dados coletados em junho de 2011 com 27 mil pessoas com mais de quinze anos em 27 países. Disponível em: <http://ec.europa.eu/public_opinion/archives/ebs/ebs_372_en.pdf>. Acesso em: $1^{\circ}$ ago. 2012.

5 Pesquisa "Extreme Weather, Climate \& Preparedness in the American Mind", realizada pela Yale Project on Climate Change Communication e pela George Mason University Center for Climate Change Communication. Dados coletados em março de 2012 com 1.008 norte-americanos com dezoito anos ou mais. Disponível em: $<$ http://environment.yale.edu/climate/files/Six-Americas-March-2012.pdf>. Acesso em: $1^{\circ}$ ago. 2012.

6 Pesquisa Global Online Environmental Survey, realizada pela Nielsen. Dados coletados em março e abril de 2011 com 25 mil consumidores em 51 países. Disponível em: <http://www.nielsen.com/content/dam/corporate/us/en/reports-downloads/2011-Reports/nieslen-sustainability-report.pdf $>$. Acesso em: $1^{\circ}$ ago. 2012. 
7 Carta Aberta ao Brasil sobre Mudanças Climáticas assinada por 22 empresas durante o Seminário "Brasil e as Mudanças Climáticas: Oportunidades para uma Economia de Baixo Carbono", em 25 de agosto de 2009. O documento é uma iniciativa do Instituto Ethos, Vale e Fórum Amazônia Sustentável. O evento foi organizado pelo Valor Econômico e Globonews, com apoio do Instituto Ethos.

8 Estudo Radar desenvolvido pela rede GlobeScan em 2011 em 21 países.

9 Em cada país são realizadas de 500 a 1.250 entrevistas. As amostras de Brasil, China, Filipinas, Indonésia e Turquia foram exclusivamente urbanas, representando entre $18 \%$ e $56 \%$ da população total.

10 Dimensão estrutural: PIB e PIB per capita (Fundo Monetário Internacional); dimensão institucional: Índice de Democracia (Economist Intelligence Unit) e Índice de Desenvolvimento de Tecnologia de Informação e Comunicação (International Telecommunication Union); dimensão ambiental: Índice de Poluição (World Health Organization), Índice de Risco Global e seus subindicadores (Bündnis Entwicklung Hilft), emissão de dióxido de carbono per capita (United Nations Development Programme), ocorrência de desastres naturais (Centre for Research on the Epidemiology of Disasters), Índice de Vulnerabilidade Ambiental (South Pacific Applied Geoscience Commission e United Nations Environment Programme) e Índice de Sociedade Sustentável e seus subindicadores (Sustainable Society Foundation).

11 O teste de Breusch e Pagan confirma a escolha pelo modelo de efeitos aleatórios.

$12 \mathrm{O}$ indicador da quantidade de empresas publicando relatórios no formato GRI foi incorporado no modelo também em sua forma quadrática devido à relação não linear apresentada com a variável dependente.

\section{Referências}

ADGER, W. N. et al. Are there social limits to adaptation to climate change? Climatic Change, v.93, n.3-4, p.335-54, 2009.

BAUER, M.; DURANT, J.; EVANS, G. European public perceptions of science. International Journal of Public Opinion Research, v.6, n.2, p.163-86, 1994.

BECK, U. Risk society: towards a new modernity. London: Sage, 1992.

BERIAIN, J. (Comp.) Las consecuencias perversas de la Modernidad. Barcelona: Anthropos, 1995.

BERMANN, C. Economía verde: una vía para otro mundo posible? Nueva Sociedad, v.239, p.11-23, May-Jun, 2012.

BLOCK, B. Covering climate change. World Watch Magazine, Washington, DC, v.23, n.2, Mar.-Apr. 2010.

BOYKOFF, M. T.; MANSFIELD, M. "Ye olde hot aire": reporting on human contributions to climate change in the UK tabloid press. Environmental Research Letters, n.3, 2008.

BROWN, L. Climate change means less food, more hunger. The Washington Post, Nov. 22,2009 . 
BURCH, S. Sustainable development paths: investigating the roots of local policy responses to climate change. Sustainable Development, v.19, n.3, p.176-88, May.-Jun. 2011.

CHAN, N.; PARKER, D. Response to dynamic flood hazard factors in peninsular Malaysia. The Geographic Journal, v.162, n.3, p.313-25, Nov. 1996.

DOSMAN, D.; ADAMOWICZ, W. L.; HRUDEY, S. Socioeconomic determinants of health - and food safety - related risk perception. Risk Analysis, v.21, n.2, p.307-17, Apr. 2001.

DOW, K. Exploring the differences in our common future(s): the meaning of vulnerability to global environmental change. Geoforum, v.23, n.3, p.417-36, 1992.

FANKHAUSER, S.; TOL, R. S. J. The social costs of climate change: the IPCC second assessment report and beyond. Mitigation and Adaptation Strategies for Global Change, v.1, n.4, p.385-403, 1997.

GIDDENS, A. A política da mudança climática. Rio de Janeiro: Zahar. 2010.

GLOBESCAN. Radar - Database of polled citizen's attitudes in 20-30 country of over fifteen years towards business and its role in society, 2011. Disponível em: <http:// www.globescan.com/expertise/trends/globescan-radar.html>.

GOUREVITCH, P. Democracy and economic policy: elective affinities and circumstantial conjunctures. World Development, v.21, n.8, p.1271-80, Aug. 1993.

GRAHAM, J. D.; CHANG, B.; EVANS, J. S. Poorer is riskier. Risk Analysis, v.12, n.3, p.333-7, Sep. 1992.

HERTIN, J. et al. Business and climate change: measuring and enhancing adaptive capacity, Progress report: Preliminary results from the house building and water sector. Brighton: SPRU/Tyndall Centre for Climate Change Research, 2002.

HOFFMAN, A. J. Carbon strategies: how leading companies are reducing their climate change footprint. Ann Arbor, MI: University of Michigan Press, 2007.

HOLlOWAY, P. Carbon dioxide capture and geologic storage. Phil. Trans. R. Soc. A, v.365, n.1853, p.1095-2107, Apr. 2007.

JACKSON, T. Motivating sustainable consumption - a review of evidence on consumer behavior and behavioural change. In: A report to the sustainable development research network. Guildford: Centre for Environmental Strategies, University of Surrey, 2005.

Prosperity Without Growth? The Transition to a Sustainable Economy. London: Earthscan, 2009.

KIM, S.; CHOI, S. Individual perception vs. structural context: searching for multilevel determinants of science-technology acceptance across 34 countries. (Under Review) 2011. Disponível em: <http://ajou.ac.kr/ seoyong/paper/Individual\%20perception $\% 20$ vs. $\% 20$ structural $\% 20$ context-with\%20Changok\%20Choi.pdf>. Acesso em: $1^{\circ}$ ago. 2012.

LORD, C. G.; ROSS, L.; LEPPER, M. R. Biased assimilation and attitude polarization: the effects of prior theories on subsequently considered evidence. Journal of Personality and Social Psychology, v.37, n.11, p.2098-109, Nov. 1979.

LUND, H. Renewable energy strategies for sustainable development. Energy, v.32, n.6, p.912-19, Jun.2007. 
MONT, O. et al. Exploring pathways towards sustainable lifestyles 2050. In: GLOBAL RESEARCH FORUM ON SUSTAINABLE CONSUMPTION AND PRODUCTION WORKSHOP, 2012, Rio de Janeiro/RJ. Anais... Rio de Janeiro: Global Research Forum on SCP, 2012. Disponível em: <http://grfscp.files.wordpress. com/2012/05/grf-2012-rio-mont-et-al.pdf>. Acesso em: 6 ago. 2012.

O'BRIEN, K. L. et al. Questioning complacency: climate change impacts, vulnerability, and adaptation in Norway. Ambio, v.35, n.2, p.50-6, Mar. 2006.

O'RIORDAN, T.; JORDAN, A. Institutions, climate change and cultural theory: towards a common analytical framework. Global Environmental Change, v.9, n.2, p.8193, 1999.

RACHLINSKI, J. J. The psychology of global climate change. University of Illinois Law Review, v.2000, n.1, p.299-319, 2000.

RAJAN, S. C. Climate change dilemma: technology, social change, or both? An examination of long-term transport policy choices in the United States. Energy Policy, v.34, n.6, p.664-79, Apr. 2006.

RAYNER, S.; MALONE, E. L. (Ed.) Human Choice and Climate Change. Volume 3: The Tools for Policy Analysis. Columbus, OH, USA: Battelle Press, 1998.

SCHERAGA, J.; GRAMBSCH, A. Risks, opportunities, and adaptation to climate change. Climate Research, v.10, n.1, p.85-95, 1998.

SLOVIC, P. Trust, emotion, sex, politics, and science: surveying the risk-assessment battlefield. Risk Analysis, v.19, n.4, p.689-701, Aug. 1999.

SMITH, J. B.; LENHART, S. S. Climate change adaptation policy options. Climate Research, v.6, n.2, p.193-201, 1996.

SMIT, B.; PILIFOSOV, O. Adaptation to climate change in the context of sustainable development and equity. In: MCCARTHY, J. J. et al. (Ed.) Climate Change 2001: impacts, adaptation and vulnerability. Cambridge: Cambridge University Press, 2001. p.877-912.

UNEP. Global Green New Deal: Policy Brief. United Nations Environment Programme, Mar. 2009.

WBCSD. Vision 2050: The New Agenda for Business. World Business Council for Sustainable Development, Feb. 2010.

WEBB, J. Climate change and society: the chimera of behaviour change technologies. Sociology, v.46, n.1, p.109-25, Feb. 2012.

WHITMARSH, L. Are flood victims more concerned about climate change than other people? The role of direct experience in risk perception and behavioural response. Journal of Risk Research, v.11, n.3, p.351-74, 2008.

WINKLER, H.; MARQUARD, A. Changing development paths: from an energy-intensive to low-carbon economy in South Africa. Climate and Development, v.1, n.1, p.47-65, 2009. 
RESUMO - O trabalho tem como objetivo identificar a percepção da opinião pública internacional diante das principais alternativas de resposta às mudanças climáticas: inovação tecnológica versus mudanças no estilo de vida individual; e os determinantes por trás dessa posição. A investigação utiliza dados em painel para quinze países, coletados em três unidades de tempo. Os resultados mostram um predomínio da escolha por soluções tecnológicas para resolver o problema, especialmente nos países emergentes, mas tal percepção vem enfrentando queda nos últimos anos, cedendo espaço para aceitação da opção por mudanças no comportamento individual. São as nações que possuem condições ambientais menos favoráveis, maior desenvolvimento humano e forte desenvolvimento institucional as que tendem a ver como menos efetivas as soluções tecnológicas para o problema das mudanças climáticas.

PALAVRAS-CHAVE: Mudanças climáticas, Tecnologia, Comportamento, Opinião pública. ABSTRACT - This study aims to explore how international public opinion perceives the main options for solving the climate change issue, technological innovations or lifestyle changes, and in what way contextual forces help to understand the prevailing choice among the publics. Research is based on random-effects, pooled time series data panel for 15 countries across 3 times points between 2007 and 2011, compiling public opinion survey data and macro-level data. Results indicate favoritism for a technology fix, especially among developing societies. However, such option is gradually losing ground and the belief in individual behavior changes is increasing. Structural, institutional and environmental forces play a role in shaping perceptions in such a way that less favorable environmental conditions coupled with higher human and institutional development motivate lower reliance in technological solutions.

KErWORDs: Climate change, Technology, Behavior, Public opinion.

Fabián Echegaray é Ph.D em Ciência Política pela Universidade de Connecticut (Estados Unidos). Atualmente é diretor geral do Instituto Market Analysis.

@ - fabian@marketanalysis.com.br

Michele H. F. Afonso é estatística pela Universidade Federal do Rio Grande do Sul, mestre em Engenharia de Produção pela Universidade Federal de Santa Catarina e pesquisadora do Instituto Market Analysis. @ - michele@marketanalysis.com.br

I, II Instituto Market Analysis, Florianópolis/SC, Brasil. 


\section{Anexo}

Descrição dos indicadores secundários utilizados neste trabalho.

IDH - O Índice de Desenvolvimento Humano é uma medida social desenvolvida pela Organização das Nações Unidas (ONU) com o objetivo de identificar o grau de progresso social atingido em cada país. O índice é composto a partir de dados de expectativa de vida ao nascer, educação e PIB per capita. A variação do índice é de 0 a 1 , em que 0 indica baixo desenvolvimento humano e 1 indica alto desenvolvimento humano.

GRI - A medida que chamaremos de GRI se refere à quantidade de empresas que determinada nação possui publicando relatórios de sustentabilidade no formato determinado pelo Global Reporting Initiative, indicando, assim, uma métrica do alinhamento das instituições com os valores de sustentabilidade. A medida é uma contagem de empresas, podendo variar de 0 até a quantidade máxima de empresas de um país, sendo que, quanto maior a medida maior é o alinhamento institucional do país com os valores de sustentabilidade.

FREE - O indicador de liberdade (Freedom index) é calculado pela fundação The Heritage Foundation em parceria com o The Wall Street Journal. O indicador é construído com base em dez medidas de liberdade econômica, as quais podem ser agrupadas em quatro pilares: estado de direito (direitos de propriedade, existência de corrupção); limitações governamentais (liberdade fiscal, gastos do governo); eficiência de regulação (liberdade empresarial, liberdade de trabalho, liberdade monetária); e abertura do mercado (liberdade de negociação, liberdade de investimento, liberdade financeira). $\mathrm{O}$ indicador varia de $0 \mathrm{a}$ 100, e 100 indica liberdade máxima.

EPI - O indicador de desempenho ambiental (Environmental Performance Index), calculado pela Universidade de Yale, é composto por 22 medidas, incluindo métricas de saúde ambiental (mortalidade infantil e condições do ar e da água para a saúde humana) e de vitalidade do ecossistema (qualidade do ar, fontes de água, biodiversidade, agricultura, florestas, condições de pesca e condições do clima e energia). O indicador varia de 0 a 100, e 100 indica desempenho ambiental máximo. 